IDDF2021-ABS-0099 EXPLORING THE EFFECTS OF ACUPUNCTURE THERAPY IN RESTORING HEALTH VIA MODULATION OF INTESTINAL MICROBIOTA

${ }^{1}$ Loh Teng-Hern Tan*, ${ }^{2}$ Vengadesh Letchumanan, ${ }^{2}$ Jodi Woan-Fei Law, ${ }^{2}$ Hooi-Leng Ser, ${ }^{3}$ Bey-Hing Goh, ${ }^{2}$ Learn-Han Lee. 'Clinical School Johor Bahru, Jeffrey Cheah School of Medicine and Health Sciences, Monash University Malaysia, Malaysia; ${ }^{2}$ Novel Bacteria and Drug Discovery Research Group (NBDD), Microbiome and Bioresource Research Strength, Jeffrey Cheah School of Medicine and Health Sciences, Monash University Malaysia, Malaysia; ${ }^{3}$ Biofunctional Molecule Exploratory Research Group (BMEX), School of Pharmacy, Monash University Malaysia, Malaysia

10.1136/gutjnl-2021-IDDF.36

Background Acupuncture has been an important complementary and alternative therapy in China. It is a nonpharmacologic modality that involves penetrating the skin at specific points using the tips of thin stainless steel needles, with broad applications, safe, economical, convenient and few side effects. The procedure can be performed by manual manipulation or electrical stimulation (electroacupuncture (EA)). Recently, an increasing number of studies indicated that acupuncture/EA could modulate the gut microbiota composition, suggesting acupuncture/EA can treat diseases associated with intestinal microbial dysbiosis. Therefore, this study explores and sheds light on the existing evidence of the acupuncture/EA treatment in ameliorating various diseases by modulating intestinal microbiota composition.

Methods Based on the PRISMA guidelines, several databases (PubMed, Web of Science, ProQuest) were accessed to perform systematic literature searches using 'Acupuncture' OR 'Electroacupuncture' AND 'microbiome' as the MeSH terms. Studies reporting on the health ameliorative effect of acupuncture mediated by gut microbiome modulation were included. Meanwhile, studies without the involvement of gut microbiome modulation were excluded, along with reviews, conference proceedings and commentaries.

Results Thirty-two studies in accordance with the inclusion criteria out of 446 articles were selected for qualitative analysis. Eight out of the 32 analysed studies involve human clinical studies that demonstrated acupuncture/EA modulates gut microbiota composition, resulting in improvement of several clinical conditions, including obesity, irritable bowel syndrome, Crohn's disease, migraine and hypertension. The remaining 24 studies evaluated the efficacy and mechanism of acupuncture/EA in ameliorating diseases linked to gut dysbiosis in rodent models, such as cancer, metabolic-, gastrointestinal-, and neurological-related disorders. Essentially, these studies observed that the therapeutic effects of acupuncture/ EA treatment were achieved by restoring the intestinal microbial balance, ameliorating intestinal barrier function and modulating the intricate interplay between intestinal microbiota, gut-brain axis and the host immune system (IDDF2021-ABS0099 Figure 1).

Conclusions In short, these studies highlighted the therapeutic effects of acupuncture/EA in improving various clinical problems by restoring the host-gut microbiota interactions. Nonetheless, future studies should clarify the exact mechanism by which acupuncture/EA modulates the gut microbiota while considering the acupoint specificity, acupoint combination and different intervention measures.

\section{IDDF2021-ABS-0101 GUT MICROBIOME DIVERSITY MEASURES FOR METABOLIC CONDITIONS: A SYSTEMATIC SCOPING REVIEW}

Chatpol Samuthpongtorn*, Tanawin Nopsopon, Krit Pongpirul. Chulalongkorn University Faculty of Medicine, Thailand

\subsection{6/gutjnl-2021-IDDF.37}

Background Evidence on the association between the gut microbiome and metabolic conditions has been increasing during the past decades. Unlike the straightforward identification of beneficial non-pathogenic bacteria as a potential probiotic for clinical use, the analysis of gut microbiome diversity is more complex and required a better understanding of various
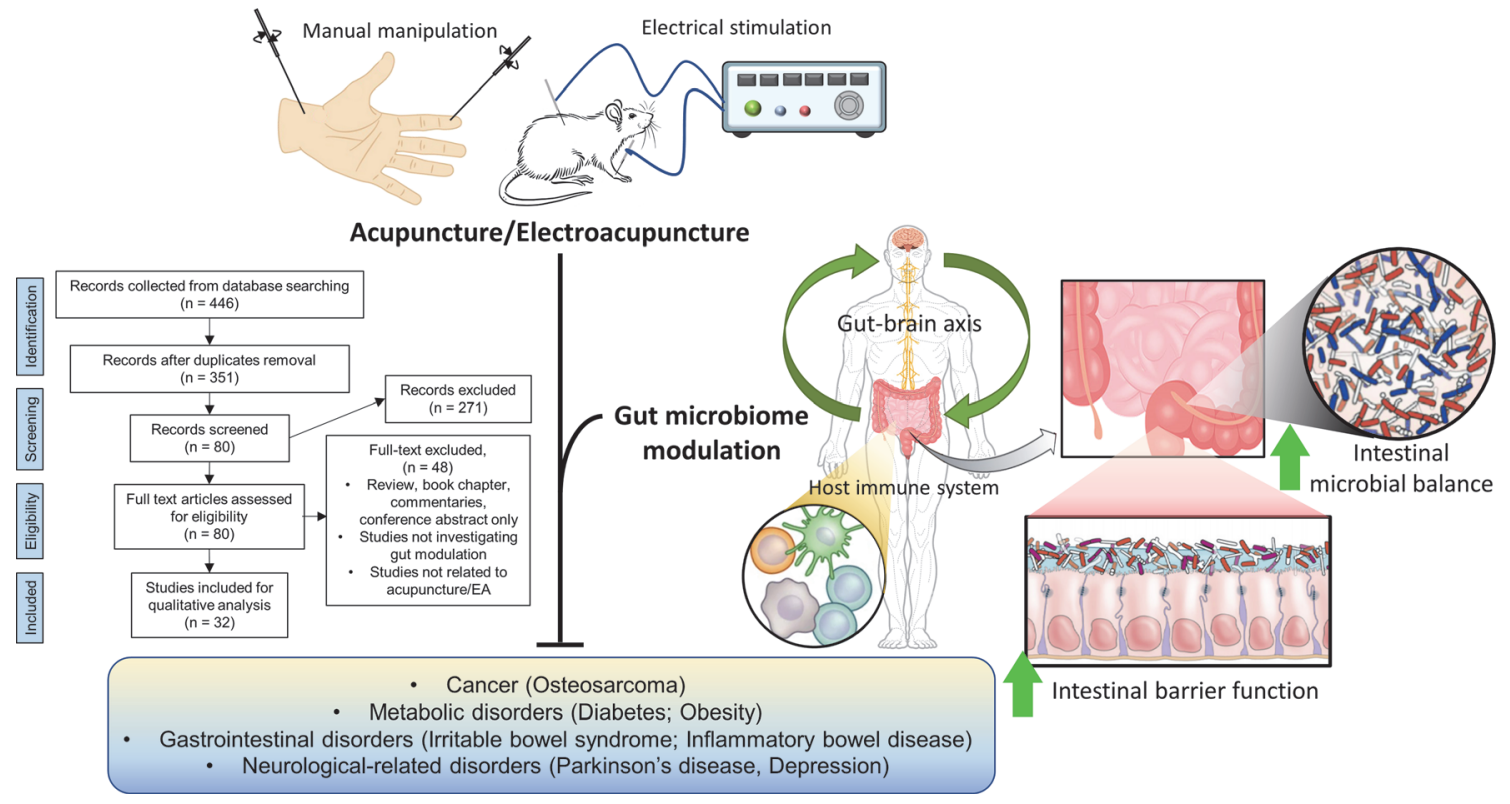
measures. We aim to summarize an elaborated list of gut microbiome diversity measures.

Methods Systematic search was conducted in three databases: PubMed, Embase, and Cochrane Central Register of Clinical Trials for randomized controlled trials, quasi-experimental and observational studies for the relationship between gut microbiota and metabolic diseases published in 2019 with the English language.

Results The measurement methods of alpha diversity and beta diversity were explored. Of 5929 potential studies, 47 were included in the systematic review (14632 patients). Of 13 alpha diversity measures, the Shannon index was the most commonly used in 37 studies (78.7\%), followed by Chao1 index (19 studies), Operational Taxonomic Unit (OTU) richness (15 studies), Simpson index (13 studies), and Abundance-based Coverage Estimators (ACE) index (10 studies). Of 2 beta diversity measures, the UniFrac was the most commonly used in 24 studies (unweighted 17 studies and weighted 16 studies), followed by Bray-Curtis dissimilarity (16 studies).

Conclusions Various measurements of gut microbiome diversity have been used in the literature. All measurements have unique characteristics, advantages, and disadvantages which lead to different usage frequencies. The measures were chosen considering cost, simplicity, and types of research.

\section{IDDF2021-ABS-0106 SIGNIFICANCE OF THE EXPRESSION OF IRON DEATH-RELATED GENE HSBP1 IN ESOPHAGEAL SQUAMOUS CELL CARCINOMA}

Mingxin Zhang ${ }^{*}$, Ning Lu, Qian Li, Manli Cui. The First Affiliated Hospital of Xi'an Medical University, China

\subsection{6/gutjnl-2021-IDDF.38}

Background To analyze the prognosis and expression of the ferroptosis-related gene HSBP1 in esophageal squamous cell carcinoma and its possible regulatory mechanisms.
Methods Screen ferroptosis-related genes from previously published literature and GSEA (Gene Set Enrichment Analysis) website. We used R 4.0.3 software to perform differential analysis on the expression and prognosis of ferroptosis-related genes, and to evaluate the immune score.

Results A total of 30 ferroptosis-related genes were differentially expressed in esophageal squamous cell carcinoma (IDDF2021-ABS-0106 Figure 1, IDDF2021-ABS-0106 Figure 2). Prognostic analysis showed that only HSBP1 was associated with the prognosis of esophageal squamous cell carcinoma, and its high expression indicated a poor prognosis (IDDF2021-ABS-0106 Figure 3). In addition, univariate and multivariate COX regression analysis further proved that high expression of HSBP1 is an independent risk factor affecting prognosis (IDDF2021-ABS-0106 Table 1). GSEA enrichment

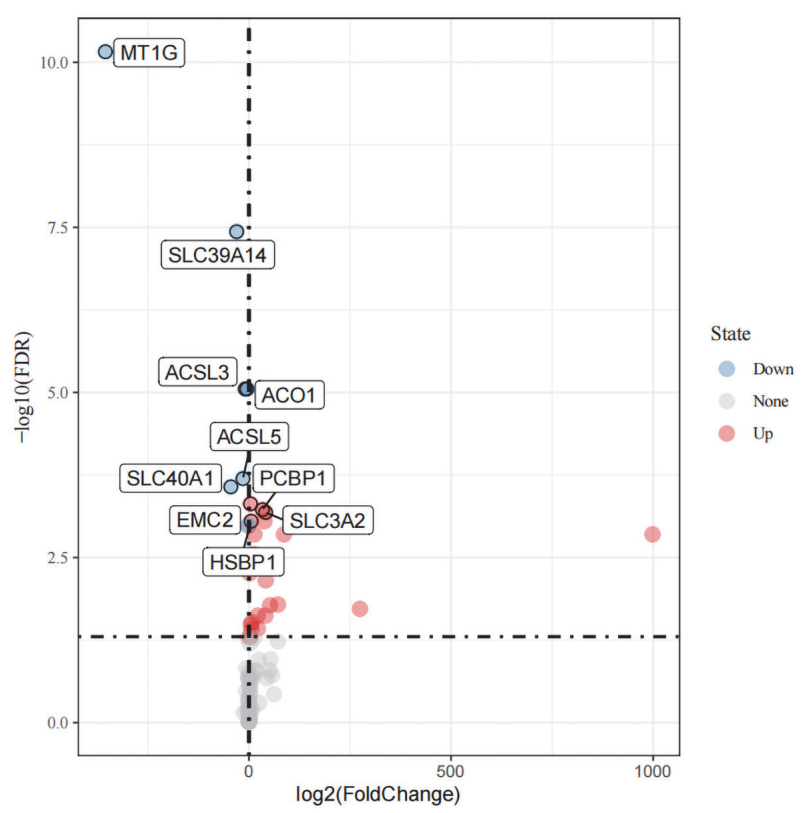

Abstract IDDF2021-ABS-0106 Figure 2

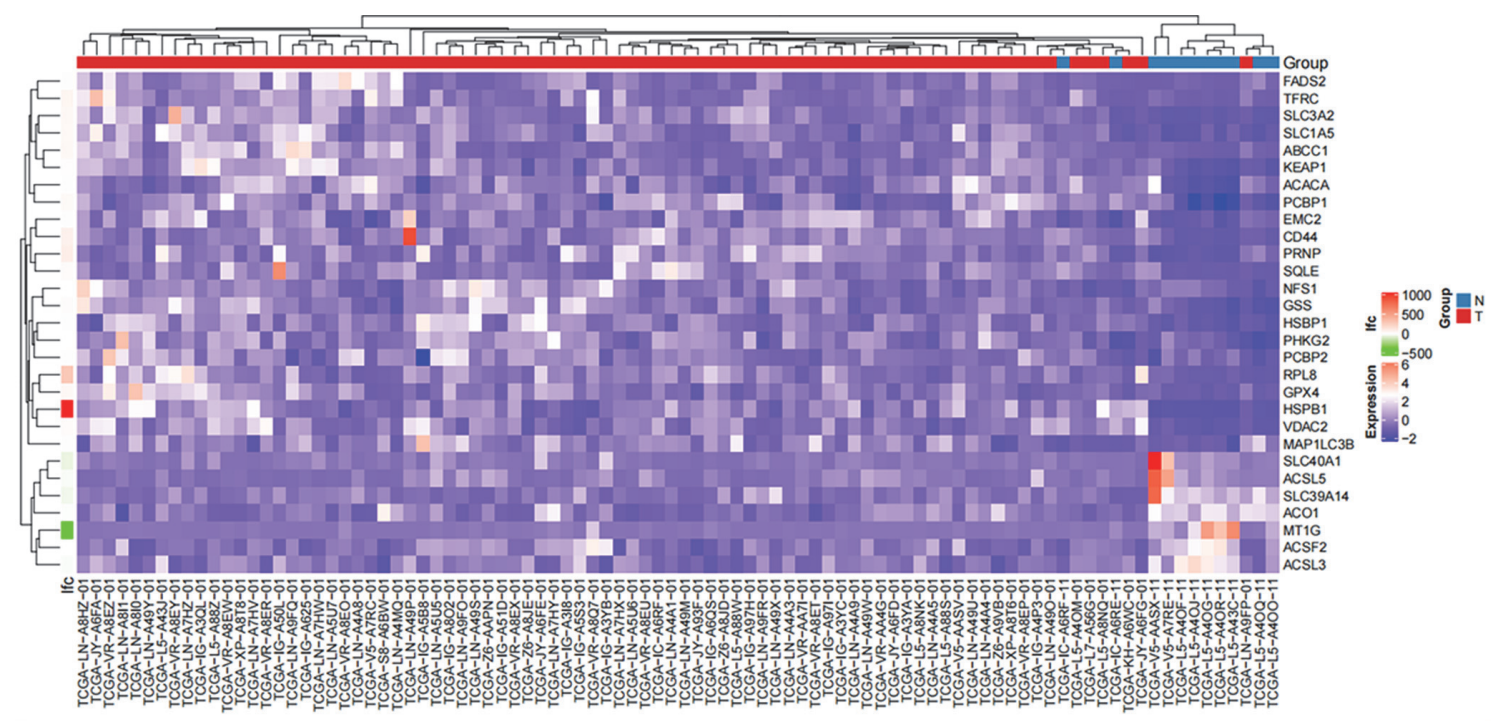

